\title{
CONOCIMIENTO Y ACEPTABILIDAD DE ANALGESIA EPIDURAL PARA EL TRABAJO DE PARTO EN EL INSTITUTO NACIONAL MATERNO PERINATAL -2011
}

\author{
Celina Montes Ruiz¹, Rocío Dávila Román²
}

\begin{abstract}
RESUMEN
Objetivos. Determinar el nivel de conocimiento y aceptabilidad de la gestante a la analgesia de parto epidural por intervención del médico anestesiólogo en el Instituto Nacional Materno Perinatal. Materiales y métodos. Estudio observacional, prospectivo, longitudinal realizado en el servicio de psicoprofilaxis del Instituto Nacional Materno Perinatal, realizado a 205 gestantes. Resultados. El nivel de conocimiento de la gestante sobre la analgesia de parto epidural por intervención del médico anestesiólogo en el Instituto Nacional Materno Perinatal es de 55,9\%, su nivel de aceptación es de 42,9\%, los Motivos (factores) que disminuyen la aceptación de la analgesia de parto son: Tener miedo de sufrir algún daño a su salud $(67,8 \%)$ y Tener miedo al daño a su bebé $(32,2 \%)$. Por último, el porcentaje de gestantes satisfechas con la Información que ha recibido es el $69,3 \%$, pero sólo el $42,4 \%$ si admite que quisiera recibir analgesia epidural en su parto, mientras que el $26,8 \%$ no quiere recibirla o tiene duda para recibirla. Conclusión. El nivel de conocimiento de la gestante es $55,9 \%$ y aceptabilidad $42,9 \%$ sobre la analgesia de parto epidural por intervención del médico anestesiólogo en el Instituto Nacional Materno Perinatal.
\end{abstract}

Palabras claves: Conocimiento; Aceptabilidad; Analgesia Epidural; Trabajo de Parto(fuente: DeCS BIREME).

\section{KNOWLEDGE AND ACCEPTABILITY OF EPIDURAL ANALGESIA FOR LABOR ON MATERNAL PERINATAL NATIONAL INSTITUTE- 2011}

\begin{abstract}
Objectives. Determine the level of awareness and acceptability of pregnant women to epidural labor analgesia anesthesiologist intervention in the Maternal Perinatal Specialized Institute. Materials and methods. This is a Observational, prospective, longitudinal study in the service of psycho-prophylaxis of the Maternal Perinatal Specialized Institute, conducted at 205 pregnant women. Results. The level of knowledge of pregnant women on epidural labor analgesia through of anesthesiologist intervention in the Maternal Perinatal Specialized Institute is $55,9 \%$, its level of acceptance is $42,9 \%$, the grounds (factors) that decrease the acceptance of labor analgesia are: Fear of being damaged your health $(67,8 \%)$ and fear of harming your baby $(32,2 \%)$. Finally, the percentage of pregnant women satisfied with the information you received is $69,3 \%$, but only admit you would like to receive epidural analgesia in labor an $42,4 \%$, while $26,8 \%$ do not want to receive or has doubts for receive this procedure. Conclusion. The level of knowledge of pregnant women is $55,9 \%$ and $42,9 \%$ acceptability of epidural labor analgesia for anesthesiologist intervention in Maternal Perinatal National Institute.
\end{abstract}

Key words: Knowledge, Acceptability; Epidural Analgesia; Labor (source: MeSH NLM).

\section{INTRODUCCIÓN}

Aunque la intensidad del dolor en el trabajo de parto varía mucho de una mujer a otra, numerosos estudios demuestran que la mayor parte de ellas, lo califican como severo y su presencia constituye una clara indicación para el empleo de técnicas analgésicas, más allá, de cualquier consideración de tipo médico.

Debido a todos los cambios que origina el dolor obstétrico tanto en la madre como en el producto, tenemos argumento suficiente para enfatizar que es necesario y esencial el control del dolor en la parturienta. Hoy en día se acepta unánimemente que el bloqueo epidural es el método más eficaz y seguro de analgesia para el trabajo de parto.
La fuente principal de información para el paciente continúa siendo el médico y el personal sanitario pero cada vez existen más fuentes de información que el paciente utiliza y tiene a su disposición para el cuidado de su salud, tales como, los medios de comunicación, los folletos informativos, internet y las asociaciones de pacientes. La información es un deber médico y no solamente desde el punto de vista ético o como instrumento para mejorar la relación con el paciente sino también legal. A pesar de la popularización del uso de ésta técnica paliativa del dolor durante el parto, en los últimos años se escuchan voces contrarias a la utilización de la analgesia epidural. Algunos profesionales de la salud discrepan acerca de las ventajas de esta práctica, advirtiendo de los inconvenientes que su utilización plantea, tanto para la mujer como para el feto. Por

\footnotetext{
Médico Anestesiólogo. Magíster en Salud Pública, Jefe del Departamento de Anestesia, Analgesia y Reanimación, Instituto Nacional Materno Perinatal. Lima-Perú.

2 Médico Anestesiólogo. Magíster en Gerencia en Servicios de Salud, Médico Residente de la Sub-especialidad de Anestesiología Obstétrica, Instituto Nacional Materno Perinatal. Lima-Perú.
}

Recibido: 10-10-12 Aprobado: 22-04-13 
tanto la gestante como paciente activo reclama un nuevo modelo de ejercicio profesional sanitario, que proporcione una información que les permita participar en el proceso de toma de decisiones en lo que respecta a su parto.

\section{MATERIAL Y MÉTODOS}

\section{VARIABLES DE ESTUDIO}

\section{Independientes:}

- Aceptabilidad de analgesia de parto por información brindada por el médico anestesiólogo

- Nivel de conocimiento de analgesia de parto

Dependientes:

- Paridad de la gestante

- Satisfacción de la gestante frente a la charla realizada

Intervinientes:

- Edad

- Grado de Instrucción

- Estado civil

- Numero de gestaciones

\section{METODOLOGÍA}

Tipo de investigación: Estudio observacional, prospectivo, longitudinal. Se realizó en el Servicio de psicoprofilaxis del Instituto Nacional Materno Perinatal.

\section{POBLACIÓN}

\section{Criterios de inclusión}

- Todo gestante que acuda a la charla de psicoprofilaxis.

- Paciente que lea y escriba.

\section{Criterios de exclusión}

- Paciente que no acepte participar del estudio.

- Paciente analfabeta.

\section{MUESTRA}

Unidad de Análisis: gestantes que acuden al servicio de psicoprofilaxis que cumplan con los criterios de inclusión. Tipo de muestreo: no probabilística por conveniencia.

Tamaño Muestral: la muestra para el presente estudio se tomó en base a todas las gestantes de psicoprofilaxis previo consentimiento informado.

\section{RESULTADOS}

Las características de la población de estudio son: Gestantes con edad promedio de 24,2 $\pm 6,7$ (14-39) años, en su mayoría de religión católica $(92,7 \%)$, estado civil soltera (39\%), educación secundaria $(56,1 \%)$. La ocupación principal es dedicado a "su casa" (51,2\%),
Tabla 1. Características Sociodemográficas de las gestantes atendidas en el Instituto Nacional Materno Perinatal. 2011

\begin{tabular}{|c|c|c|c|}
\hline \multicolumn{2}{|c|}{ Datos Sociodemográficos } & \multirow{2}{*}{$\frac{\mathbf{N}}{190}$} & \multirow{2}{*}{$\begin{array}{c}\% \\
92,7 \%\end{array}$} \\
\hline \multirow{2}{*}{ Religión. } & Católica & & \\
\hline & Cristiana & 15 & $7,3 \%$ \\
\hline \multirow{3}{*}{ Estado Civil. } & Soltera & 80 & $39,0 \%$ \\
\hline & Conviviente & 75 & $36,6 \%$ \\
\hline & Casada & 50 & $24,4 \%$ \\
\hline \multirow{3}{*}{$\begin{array}{l}\text { Grado de Ins- } \\
\text { trucción. }\end{array}$} & Primaria & 0 & $.0 \%$ \\
\hline & Secundaria & 115 & $56,1 \%$ \\
\hline & Superior & 90 & $43,9 \%$ \\
\hline \multirow{3}{*}{ Ocupación. } & Ama de Casa & 105 & $51,2 \%$ \\
\hline & Estudiante & 65 & $31,7 \%$ \\
\hline & Trabaja & 35 & $17,1 \%$ \\
\hline \multirow{3}{*}{$\begin{array}{l}\text { Número de } \\
\text { parto. }\end{array}$} & Primer & 170 & $82,9 \%$ \\
\hline & Segundo & 30 & $14,6 \%$ \\
\hline & Tercer o más & 5 & $2,4 \%$ \\
\hline \multicolumn{2}{|c|}{ Total } & 205 & $100,0 \%$ \\
\hline
\end{tabular}

siendo mayormente primíparas $(82,9 \%)$ (Tabla 1). El $64,4 \%$ de las gestantes son residentes de San Juan de Lurigancho, Cercado de Lima y la Victoria (Tabla 2).

Las características de la población de estudio son:

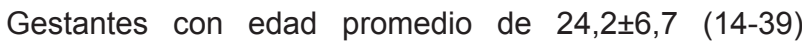
años, en su mayoría de religión católica $(92,7 \%)$, estado civil soltera $(39 \%)$, educación secundaria $(56,1 \%)$. La ocupación principal es dedicado a "su casa" (51,2\%), Victoria (Tabla 2).

Respecto a la información sobre Analgesia epidural en el parto, en términos generales $44(21,5 \%)$ no contestaron la pregunta, por lo que con el $78,5 \%$ de los encuestados podemos describir que el $62,7 \%$ de las gestantes si sabe o a escuchado hablar sobre analgesia epidural en el parto y el $67,3 \%$ ha recibido la información de un profesional de la salud. El $94,1 \%$ no ha recibido analgesia epidural en el parto y un $57,1 \%$ no aceptaría su colocación (Tabla 3 ).

Tabla 2. Procedencia de las de las gestantes atendidas en el Instituto Nacional Materno Perinatal. 2011

\begin{tabular}{lrr}
\hline Procedencia & N & \multicolumn{1}{c}{$\%$} \\
\hline San Juan de Lurigancho & 76 & $37,1 \%$ \\
\hline Cercado de Lima & 30 & $14,6 \%$ \\
\hline La Victoria & 26 & $12,7 \%$ \\
\hline El Agustino & 15 & $7,3 \%$ \\
\hline San Martin de Porres & 14 & $6,8 \%$ \\
\hline Breña & 12 & $5,9 \%$ \\
Jesús María & 7 & $3,4 \%$ \\
Ate & 5 & $2,4 \%$ \\
Callao & 5 & $2,4 \%$ \\
Chorrillos $\quad 5$ & $2,4 \%$ \\
Independencia & 5 & $2,4 \%$ \\
\hline Puente piedra & 5 & $2,4 \%$ \\
\hline
\end{tabular}


Tabla 3. Información sobre Analgesia Epidural en el Parto, de las gestantes atendidas en el Instituto Nacional Materno Perinatal. 2011

\begin{tabular}{|c|c|c|c|}
\hline \multicolumn{2}{|c|}{$\begin{array}{l}\text { Información sobre analgesia epidural en } \\
\text { el de parto }\end{array}$} & \multirow{2}{*}{$\begin{array}{c}\mathbf{N} \\
90\end{array}$} & \multirow{2}{*}{$\begin{array}{c}\% \\
55,9 \%\end{array}$} \\
\hline \multirow{6}{*}{$\begin{array}{l}\text { Sabe sobre } \\
\text { Analgesia } \\
\text { Epidural en el } \\
\text { Parto. }\end{array}$} & Sí & & \\
\hline & No & 60 & $37,3 \%$ \\
\hline & He escuchado & 11 & $6,8 \%$ \\
\hline & Subtotal & 161 & $100,0 \%$ \\
\hline & No contestaron & 44 & - \\
\hline & Total & 205 & . \\
\hline \multirow{5}{*}{$\begin{array}{l}\text { Ha recibido } \\
\text { analgesia de } \\
\text { parto Epidural. } \\
\text { Aceptaría } \\
\text { colocación } \\
\text { analgesia de } \\
\text { parto epidural. }\end{array}$} & Sí & 12 & $5,9 \%$ \\
\hline & No & 193 & $94,1 \%$ \\
\hline & Sí & 88 & $42,9 \%$ \\
\hline & No & 117 & $57,1 \%$ \\
\hline & Total & 205 & $100,0 \%$ \\
\hline \multirow{6}{*}{$\begin{array}{l}\text { Cómo se ha } \\
\text { enterado del } \\
\text { procedimiento } \\
\text { de Analgesia de } \\
\text { parto Epidural. }\end{array}$} & $\begin{array}{l}\text { Un profesional de la salud } \\
\text { me ha informado }\end{array}$ & 138 & $67,3 \%$ \\
\hline & $\begin{array}{l}\text { Me ha contado una amiga, } \\
\text { familiar o conocido }\end{array}$ & 48 & $23,4 \%$ \\
\hline & He visto en la televisión & 35 & $17,1 \%$ \\
\hline & Vi en internet & 26 & $12,7 \%$ \\
\hline & He escuchado en la radio & 1 & $.5 \%$ \\
\hline & $\begin{array}{l}\text { Lo leí en un periódico, } \\
\text { folletos o trípticos }\end{array}$ & 1 & $.5 \%$ \\
\hline
\end{tabular}

Al $50,2 \%$ de las gestantes que escuchó la charla y les pareció que fue buena (sólo a un $8,3 \%$ le pareció inadecuada); el $69,3 \%$ refieren que si están satisfechas con la información recibida y el $62,4 \%$ desean recibir analgesia epidural en su parto (Tabla 4).

En cuanto a la decisión de recibir analgesia epidural durante el trabajo de parto, el $61,5 \%$ lo hizo por la información que recibió del personal de salud y respecto al motivo de "NO" recibir analgesia epidural en su parto, el 67,8\% menciona que es por tener miedo a sufrir daño en su salud y el otro $34,6 \%$ teme algún daño en su bebé(Tabla 5 ).

Tabla 4. Aceptabilidad de la Analgesia de Parto de las gestantes atendidas en el Instituto Nacional Materno Perinatal -2011

\begin{tabular}{lrc}
\hline Qué le pareció la charla? & N & $\%$ \\
\hline Buena & 103 & $50,2 \%$ \\
\hline Regular & 85 & $41,5 \%$ \\
Mala & 17 & $8,3 \%$ \\
\hline Está satisfecha de la Información que ha & $\mathbf{N}$ & $\%$ \\
recibido? & 142 & $69,3 \%$ \\
\hline Si & 63 & $30,7 \%$ \\
\hline Si, pero sería bueno más información & 0 & $.0 \%$ \\
\hline No la entendió & $\mathbf{N}$ & $\%$ \\
Quisiera recibir analgesia epidural en su & & \\
parto? & 128 & $62,4 \%$ \\
\hline Si & 49 & $23,9 \%$ \\
\hline No & 28 & $13,7 \%$ \\
\hline No está segura, lo va a pensar $\quad$ Total & $\mathbf{2 0 5}$ & $\mathbf{1 0 0 , 0}$ \\
\hline
\end{tabular}

Tabla 5. Decisión de recibir analgesia de parto de las gestantes atendidas en el Instituto Nacional Materno Perinatal -2011

\begin{tabular}{|c|c|c|}
\hline $\begin{array}{l}\text { Porqué decidió recibir analgesia epidural en su } \\
\text { presente parto. }\end{array}$ & $\mathbf{N}$ & $\%$ \\
\hline La información que recibí de mi familia y conocidos. & 79 & $38,5 \%$ \\
\hline La información que recibí del personal de salud. & 126 & $61,5 \%$ \\
\hline $\begin{array}{l}\text { Por la información que recibí antes de venir al } \\
\text { INMP por profesionales de la salud. }\end{array}$ & 0 & $.0 \%$ \\
\hline $\begin{array}{l}\text { Por la información que tomé de otros medios } \\
\text { (radio, TV, periódicos e internet) }\end{array}$ & 0 & $.0 \%$ \\
\hline Total & 205 & 100,0 \\
\hline $\begin{array}{l}\text { Motivo por el cual no decide recibir analgesia } \\
\text { epidural en su parto. }\end{array}$ & $\mathbf{N}$ & $\%$ \\
\hline Mi familia o mi esposo se opondrían & 0 & $.0 \%$ \\
\hline Tengo miedo de sufrir algún daño a mi salud & 139 & $67,8 \%$ \\
\hline Mi religión no me lo permite & 0 & $.0 \%$ \\
\hline $\begin{array}{l}\text { Me han contado malas experiencias de la analge- } \\
\text { sia epidural }\end{array}$ & 0 & $.0 \%$ \\
\hline Tengo miedo de daño a mi bebé & 71 & $34,6 \%$ \\
\hline
\end{tabular}

Analizando la relación entre la percepción de Información con la recepción de recibir Analgesia Epidural en el trabajo de parto, utilizamos la variable sobre la "percepción sobre la charla" y la "aceptación de recibir la analgesia epidural" encontrando que no existe relación entre estas variables $(p=0,141)$, es decir, la percepción buena acerca de la charla no genera mayor frecuencia relativa de aceptar la analgesia epidural (Tabla 6). En la figura 1 se puede observar que los porcentajes son muy similares entre los que responden afirmativamente o negativamente recibir la analgesia epidural, por otro lado, para aquellos que aceptaron una "buena charla" tienen un alto porcentaje $(71,4 \%)$ que lo pensarán la aceptación de analgesia epidural (Figura 1).

Tabla 6. Relación entre percepción de Información con recepción de recibir Analgesia Epidural en el trabajo de parto de las gestantes atendidas en el Instituto Nacional Materno Perinatal -2011

\begin{tabular}{|c|c|c|c|c|c|c|c|c|c|}
\hline \multirow{3}{*}{\multicolumn{2}{|c|}{$\begin{array}{l}\text { Relación entre } \\
\text { percepción de } \\
\text { Información con } \\
\text { recepción de recibir } \\
\text { Analgesia. }\end{array}$}} & \multicolumn{8}{|c|}{ Quisiera recibir analgesia epidural en su parto } \\
\hline & & \multicolumn{2}{|r|}{ Si } & \multicolumn{2}{|r|}{ No } & \multicolumn{2}{|c|}{$\begin{array}{l}\text { No está } \\
\text { segura, lo va } \\
\text { a pensar }\end{array}$} & \multicolumn{2}{|c|}{ Total } \\
\hline & & $\mathbf{N}$ & $\%$ & $\mathbf{N}$ & $\%$ & $\mathbf{N}$ & $\%$ & $\mathbf{N}$ & $\%$ \\
\hline \multirow{3}{*}{$\begin{array}{l}\text { Qué le } \\
\text { pareció la } \\
\text { charla. }\end{array}$} & Buena & 60 & $46,9 \%$ & 23 & $46,9 \%$ & 20 & $71,4 \%$ & 103 & $50,2 \%$ \\
\hline & Regular & 58 & $45,3 \%$ & 20 & $40,8 \%$ & 7 & $25,0 \%$ & 85 & $41,5 \%$ \\
\hline & Mala & 10 & $7,8 \%$ & 6 & $12,2 \%$ & 1 & $3,6 \%$ & 17 & $8,3 \%$ \\
\hline \multirow{3}{*}{$\begin{array}{l}\text { Está } \\
\text { satisfecha } \\
\text { de la } \\
\text { Información } \\
\text { que ha } \\
\text { recibido. }\end{array}$} & $\mathrm{Si}$ & 87 & $68,0 \%$ & 35 & $71,4 \%$ & 20 & $71,4 \%$ & 142 & $69,3 \%$ \\
\hline & $\begin{array}{l}\text { Sí, pero } \\
\text { sería bueno } \\
\text { más infor- } \\
\text { mación }\end{array}$ & 41 & $32,0 \%$ & 14 & $28,6 \%$ & 8 & $28,6 \%$ & 63 & $30,7 \%$ \\
\hline & $\begin{array}{l}\text { No la } \\
\text { entendió }\end{array}$ & 0 & $.0 \%$ & 0 & $.0 \%$ & 0 & $.0 \%$ & 0 & $.0 \%$ \\
\hline \multicolumn{2}{|c|}{ Total } & 128 & $100,0 \%$ & 49 & $100,0 \%$ & 28 & $100,0 \%$ & 205 & $100,0 \%$ \\
\hline
\end{tabular}




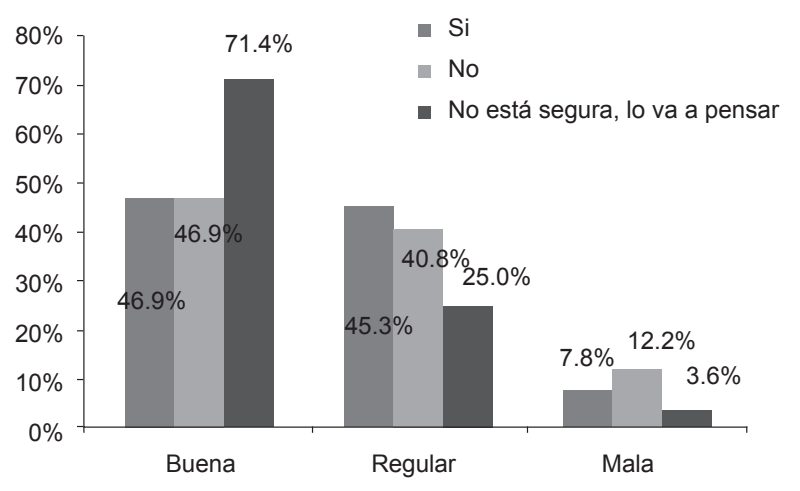

Figura 1. Relación entre percepción sobre la charla y aceptación de recibir la Analgesia Epidural en el trabajo de parto de las gestantes atendidas en el Instituto Nacional Materno Perinatal $-2011$

Analizando la variable "Satisfacción de la Información recibida." y la "aceptación de recibir la analgesia epidural" encontramos que no existe relación entre estas variables $(p=0,874)$; es decir, La respuesta afirmativa de estar satisfecho con la Información recibida no genera mayor frecuencia relativa de aceptar la analgesia epidural (Tabla 6). En la figura 2 se puede observar que los porcentajes son muy similares entre los que responden afirmativamente 0 quieren más información al respecto (Figura 2).

\section{DISCUSIÓN}

Según revisión bibliográfica de Carlos Siu titulado "Analgesia regional selectiva ambulatoria en gestantes en trabajo de parto, desarrollado en el Servicio de Obstetricia del Hospital Arzobispo Loayza entre Setiembre y Diciembre de 1998, Lima - Perú", la analgesia epidural de labor de parto es un método que permite a la paciente un significativo alivio del dolor. De los resultados encontrados en el presente estudio el nivel de conocimiento sobre estos beneficios en las gestantes es de $55,9 \%$ y un $6,8 \%$ lo ha escuchado, mientras que el

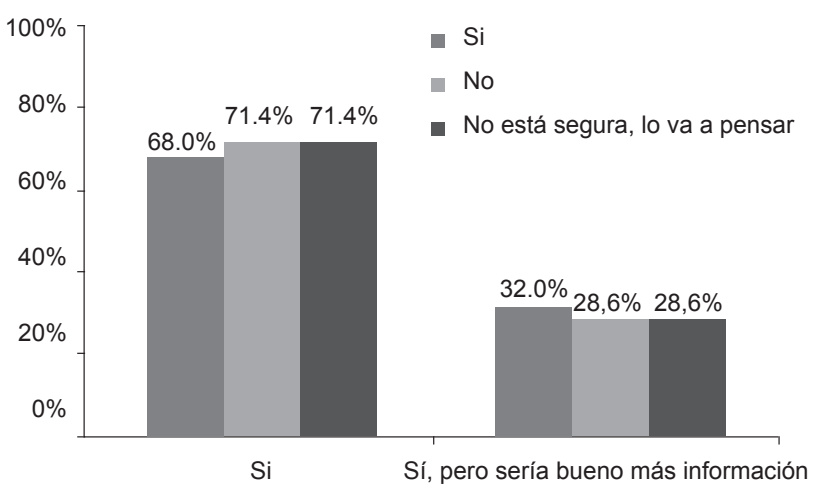

Figura 2. Relación entre la satisfacción de la Información recibida y aceptación de recibir la Analgesia Epidural en el trabajo de parto de las gestantes atendidas en el Instituto Nacional Materno Perinatal -2011
$37,3 \%$ no tiene conocimiento al respecto. Investigadores como White L, Gorton P, Wee M y colaboradores "Ventajas de la Información escrita sobre la analgesia epidural en el trabajo de parto", ofrece información escrita a las mujeres en el momento próximo al trabajo de parto el que mejora el nivel de conocimientos en relación a los beneficios, efectos adversos y riesgos de la analgesia epidural; y se postula la implementación rutinaria de esta práctica en función de la adecuada receptividad de las usuarias, lo que nos motiva a información a la gestante cuando se encuentra sin el stress del parto.

El nivel de aceptación de la gestante sobre la analgesia de parto epidural por intervención del médico anestesiólogo en el Instituto Nacional Materno Perinatal es de $42,9 \%$ y respecto a su decisión de querer recibir analgesia epidural en su parto es de $62,4 \%$. Cuyos porcentajes es bajo comparado con el valor de $92 \%$ referido en el marco referencial de Karin Rando y colaboradores en el estudio titulado: "Encuesta sobre aceptación y uso de analgesia regional para el parto, en anestesiólogos y ginecólogos", donde concluye que la población encuestada acepta la analgesia para su próximo parto. También los resultados muestran que el porcentaje de gestantes satisfechas con la Información que ha recibido es el $69,3 \%$, pero sólo el $42,4 \%$ si admite que quisiera recibir analgesia epidural en su parto, mientras que el $26,8 \%$ no quiere recibirla o tiene duda para recibirla.

Del estudio se describe que la decisión de recibir analgesia epidural durante el trabajo de parto, el $61,5 \%$ lo hizo por la información que recibió del personal de salud y el $38,5 \%$ recibió la información de la familia y de conocidos. Respecto al motivo de "NO" recibir analgesia epidural en su parto, el $67,8 \%$ menciona que es por tener miedo a sufrir daño en su salud y el otro $34,6 \%$ teme algún daño en su bebé.

El $69,3 \%$ refieren que si están satisfechas con la información recibida y como mencionamos anteriormente el $62,4 \%$ desearía recibir analgesia epidural en su parto. La referencia bibliográfica de del estudio, "Indicadores de calidad de asistencia en obstetricia" realizado por Hugo Salinas y colaboradores, en el servicio de Maternidad del Hospital Clínico de la Universidad de Chile el año 2004, considera como principal indicador de satisfacción del usuario, el número de partos con analgesia, información que a pesar de estar incorporados en los protocolos, deben ser incorporados dentro de programas con mayor información hacia la gestante.

\section{REFERENCIAS BIBLIOGRÁFICAS}

1. Siu C, Mere J, Rossi P. Analgesia Regional Selectiva Ambulatoria en gestantes en trabajo de parto. Ginecología y Obstetricia - Vol. 45 Nº 3 julio 1999. Perú.

2. Salinas $\mathrm{H}$, Erazo M y Col. Indicadores de calidad de asistencia en Obstetricia. Rev Chil Obstet Ginecol 2006; 71 (2): 114 120 Santiago 2006. 
3. White L, Gorton P, Wee M y colaboradores. Ventajas de la información escrita sobre la analgesia epidural previa al trabajo de parto. International Journal of Obstetric Anesthesia 12:09- $97-2003$.

4. Carrión B. Manual de entrevista clínica para la atención primaria de salud. Barcelona: Doyma: 1989.

5. Decálogo de los pacientes (en línea). Barcelona: Fundación Biblioteca Josep Laporte, 17 de julio de 2003.

6. Melzack R. The myth of painless childbirth. The Jonh J Bonica Lecture. Pain 1984; 19-,321-337.

7. Melzack R. Schaffelberg D. Low-back pain during labor. Am J. Obstet Gynecol 1987; 156:901-905.

8. González López-Valcárcel B. Cambio tecnológico en sanidad: determinantes, efectos y políticas. Economía de la Salud y Gestión Sanitaria. Coordinado por B. González LópezValcárcel. Editado por Masson; 2005.

9. García M. Tipología de innovaciones en servicios aplicada a la sanidad. REES 2006 Volumen 5 / Número 1.2006 / 37-40 Madrid - España.
10. Practice Guidelines for Obstetrical Anesthesia: A report by the American Society of Anesthesiologists Task Force on Obstetrical Anesthesia. Anesthesiology. 1999; 90:600-11.

11. http://www.webpacientes.org

12. http://www.fbjoseplaporte.org/dbcn

13. Kelligan P, Garza A, Jurado E. Anestesia en Ginecoobstetricia y Perinatología. PAC Anestesia 1. B-2 Primera edición 1998 Intersistemas, S.A. de C.V.

14. León Visser. Anestesia epidural. Dept. of Anesthesiology, University of Michigan Medical Center, Ann Arbor, Michigan, USA. Issue 13 (2001) Article 11: Page 1 of 4. http://www.nda. ox.ac.uk/wfsa/html/u13/u1311_01.htm

15. Cabero L, Rodríguez D. Obstetricia y medicina materno fetal. Editorial Médica Panamericana. Buenos Aires. 2007.

16. Velázquez B, García de Diego JM, Serrano del Rosal R. Análisis de la no elección de la analgesia epidural durante el trabajo de parto en las mujeres andaluzas. Rev Sociedad Española del Dolor 2010;17(1):657. http://revista.sedolor.es/ articulo.php?|D=657 